\title{
Epidemiologic Approach to the Relationship between Diabetes and Cancer: A Systematic Review Study
}

\author{
Mohammad Madmoli ${ }^{1 *}$ and Marzieh Abbaszade Aliabad ${ }^{2}$ \\ ${ }^{1}$ Student Research Committee, Shoushtar faculty of Medical Sciences, Shoushtar, Iran \\ ${ }^{2}$ Kerman University of Medical Sciences, Kerman, Iran
}

*Corresponding author: Mohammad Madmoli, Student Research Committee, Shoushtar faculty of Medical Sciences, Shoushtar, Iran

\section{ARTICLE INFO \\ Received: 栔 July 03, 2019 \\ Published: 慧 July 10, 2019 \\ Citation: Mohammad Madmoli, Marzieh Abbaszade Aliabad. Epidemiologic Ap- proach to the Relationship between Di- abetes and Cancer: A Systematic Review Study. Biomed J Sci \& Tech Res 19(3)- 2019. BJSTR. MS.ID.003313.}

Keywords: Cancer; Diabetes; Cancer Patients; Epidemiologic Approach; Relationship Between Diabetes and Cancer

\section{ABSTRACT}

Introduction: Diabetes patients have been reported to increase the risk of cancer by a large proportion than non-diabetic patients. In this study, we intend to examine the relationship between these two diseases by examining several studies. Therefore, this systematic review was conducted to determine the epidemiological approach to the relationship between diabetes and cancer.

Materials and Methods: This study was a systematic review based on the Broome method. In this study, using the papers published in the last 17 years in relation to the epidemiological approach to the relationship between diabetes and cancer. Searching articles in search engines, scientific sites and databases, Google Scholar, Embase, Sciencedirect, PubMed, in Persian and English. In the first phase, 49 articles were found. Of these, 14 articles related to the topic that were published in the last 17 years were reviewed.

Results: In this study, 14 articles were reviewed that revealed a link between diabetes and cancer and a serious risk for cancer. One of these studies showed that there was a significant relationship between type 2 diabetes and colon adenomas. Exposure to insulin and thiazolidinedione was associated with the formation of adenoma, and none of the anti-inflammatory drugs and HbA1c levels were predictive of adenoma.

Conclusion: In this study, there was a significant relationship between diabetes and various types of cancer. Therefore, it is necessary to emphasize the screening of breast cancer and other types of cancer by conducting tests and diagnostic work for the rapid diagnosis of cancer among diabetic patients. Also, diabetes has increased the risk of developing another dangerous disease, such as cancer.

\section{Introduction}

Uncontrolled diabetes mellitus is a chronic disease that affects many organs and requires care throughout the life cycle in order to limit and cope with chronic complications. It is a disorder of insulin production and function syndrome. Which led to an increase in uncontrolled outbreaks due to decreased physical activity and consumption of unhealthy foods [1-9]. The global prevalence of diabetes has increased significantly over the past two decades, from around 30 million cases in 1985 to 177 million in 2000 and based on research conducted in the year 2030 reaches over 360 million cases. And in 2014, the global prevalence of diabetes among adults older than 18 years was estimated at 9\% [10-19]. and the prevalence of this disease in Iran, according to the statistics released by the health department of the Ministry of Health in a population over the age of 30 , is more than $14 \%$ or more in the female population, and it is associated with multiple short-term and long-term complications, which in many cases is not reversible [20-38]. Among diabetic patients, depression is one of the most common psychiatric disorders. That depression is a mood that involves rage and escape from activity or unconsciousness and reluctance, which can affect one's thoughts, feelings, feelings, and well-being. This mental illness is caused by various events 
that either originated from loneliness and rejection, or from an uncontrollable and uniformity of life, in which one takes happiness and freshness from one person and makes him black and lonely.

Depression is one of the most common and debilitating problems for youth and adolescents. And depression and occupational stress may cause some disorders in the mental and physical health of individuals, and high occupational stress is known as a known psychosocial factor in the development of cardiovascular disease [39-45]. Diabetes is also one of the most common endocrine complications in thalassemic patients, and thalassemia is one of the inherited blood diseases. The disease is a genetic condition in which the red blood cells in the patient's blood do not lose their original form and cannot carry oxygen, which $\beta$-thalassemia is a group of hereditary blood disorders caused by the reduction or synthesis of the beta-hemoglobin chain of blood [46-54]. In some studies, there is a link between diabetes and cancer, and it seems that one of the complications of diabetes is the appearance of various types of cancer. Therefore, in this study, we intend to examine the relationship between these two diseases by examining several studies.

In this study, we intend to examine the relationship between these two diseases by examining several studies. Therefore, this systematic review was conducted to determine the epidemiological approach to the relationship between diabetes and cancer.

\section{Materials and Methods}

In this study, using the papers published in the last 17 years in relation to the epidemiological approach to the relationship between diabetes and cancer. It was conducted in English and Persian by searching articles in search engines, reputable scientific sites and databases, Google Scholar, Embase, Scientific Papers, Sciencedirect, PubMed. In the first phase, 49 articles were found. Of these, 14 articles related to the topic that were published in the last 17 years were reviewed. This study is a systematic review in order to achieve the goal of the study and to improve the accuracy of its study and its comprehensive understanding, this integrated overview study was conducted based on the Broome method. This method is carried out in three stages of the search of texts, data evaluation and data analysis, so that in the search phase, the texts of post-retrospective studies are examined in four stages in terms of inclusion criteria and after obtaining entry conditions The content of the study is evaluated and the data is analyzed at the end. Studies in the field of research were written in English or Persian, access to their full text was possible and published in the last 17 years, entered the study, and unnamed, unannounced and non-academic studies were deleted. To achieve relevant studies, a wide range of keywords including Cancer, Diabetes, Cancer Patients, Epidemiologic Approach, Relationship Between Diabetes and Cancer was used as a one-to-one search, combined with the method "And" and "OR".

\section{Results}

In some studies, there is a link between diabetes and cancer. Therefore, in this study, we intend to examine the relationship between these two diseases by examining several studies. In this study, we intend to examine the relationship between these two diseases by examining several studies. Therefore, this systematic review was conducted to determine the epidemiological approach to the relationship between diabetes and cancer. Studies have shown that diabetes increases the chance of different types of cancer. For example, several studies have shown that diabetes increases the risk of colorectal cancer, such as study [55], which showed that the incidence of colorectal cancer in diabetic patients was 2.1 times higher than that of non-diabetic controls, as well as in another study Which showed a 1.23 times higher chance of having colorectal cancer in diabetic patients [56].

Another study showed that diabetes was associated with an increased risk of colorectal cancer, and in this study, 207 patients with colorectal cancer had 48 patients with diabetes, compared to 207 controls, only 21 cases had diabetes [57]. The study also found that diabetes is associated with an increased risk of breast cancer, and the analysis of all 20 studies in this study showed that women with diabetes (versus diabetic men) had 20 Percentage increased risk of breast cancer [58]. Also, another study showed a significant relationship between type 2 diabetes and colon adenomas [59]. In another study, in men, the risk of developing the entire cancer in people with a history of diabetes increased by $27 \%$, as well as in women, the incidence of cancer significantly increased at the borderline, while The incidence of gastric cancer and liver cancer was statistically significant and the incidence of ovarian cancer was observed at the border [60].

In another study, the association between diabetes and primary liver cancer was investigated, of which sixty-four cases of hepatocellular carcinoma were reported in 87 diabetic patients [61]. In the study, there was no significant association between diabetes and bile, esophageal and stomach cancers, diabetes intensity, duration or need for drug therapy, it seemed that there was a stronger association with the risk of developing liver cancer, Pancreas and rectum [62]. In the study, the risk of developing pancreatic and liver cancers among diabetic patients also increased. The increased risk for pancreatic cancer was fully apparent to people with diabetes, on the contrary, increased risk of liver cancer was independent of the distance between diabetes and the diagnosis of cancer, as a result, diabetes was associated with an increased risk of developing liver cancer among men [63]. Also, in a study, diabetes mellitus was associated with an increased risk of colorectal neoplasm [64]. Of course, there was no significant relationship between diabetes and cancer in studies, for example, in the study of Dash et al., Conducted on African Americans, there was no significant relationship between diabetes and colorectal 
cancer [65]. Also, according to studies [66], there was a significant and positive correlation between high fat diet and consumption of red meat with colorectal cancer.

\section{Discussion}

Uncontrolled diabetes mellitus is a chronic disease that affects many organs and requires care throughout the life cycle in order to limit and cope with chronic complications. It is a disorder of insulin production and function syndrome. Which led to an increase in uncontrolled outbreaks due to decreased physical activity and consumption of unhealthy foods [1-9]. In some studies, there is a link between diabetes and cancer, and it seems that one of the complications of diabetes is the appearance of various types of cancer. Therefore, in this study, we intend to examine the relationship between these two diseases by examining several studies. In this study, we intend to examine the relationship between these two diseases by examining several studies. Therefore, this systematic review was conducted to determine the epidemiological approach to the relationship between diabetes and cancer.

According to a study [55-57], which showed that diabetes may increase the chances of developing colorectal cancer, colorectal cancer is a common and deadly disease with a high incidence of occurrence in various parts of the world [69] and Generally speaking, cancers can occur when parts of the body begin to grow uncontrollably, and one of the common cancers in women and men is colorectal cancer. The best way to prevent the disease is through proper nutrition and the use of fiber in nutrition, as constipation is one of the most important causes of colorectal cancer, it should be prevented. Also, according to study, which showed that diabetes was associated with an increased risk of breast cancer, it can be said that people with breast cancer at the age of menopause are more likely to have diabetes. Even statistics show that people with diabetes also have a chance of developing breast cancer, about $20 \%$ of these people develop breast cancer.

And lifestyle changes can reduce the risk of breast cancer. One of the factors that people with breast cancer are at risk for diabetes are estrogen resistance in the stages of chemotherapy. Taking some medications such as glucocorticoid in chemotherapy increases your blood sugar, taking these drugs to prevent inflammation and nausea. When the body resists insulin resistance, it is prone to spreading diabetes and a variety of cancers that can occur in people with breast cancer. Also, in diabetics, with increased levels of insulin, breast tissue changes that increase the risk of developing breast cancer. The statistics show that people with advanced breast cancer, and at the same time diabetes, have larger tumors than other breast cancer patients. In these people, the possibility of spreading the disease also increases the incidence of diabetes. Overall, it can be said that the study showed that diabetes is associated with a serious risk of developing a variety of cancers [64].

\section{Conclusion}

In this study, there was a significant relationship between diabetes and various types of cancer. Therefore, it is necessary to emphasize the screening of breast cancer and other types of cancer by conducting tests and diagnostic work for the rapid diagnosis of cancer among diabetic patients. Also, diabetes has increased the risk of developing another dangerous disease, such as cancer.

\section{Acknowledgment}

The writer of this article is grateful for all those who have had the necessary cooperation.

\section{Conflict of Interest}

There are no conflicts of interest in this article.

\section{References}

1. Moslemirad M, Madmoli M, Madmoli Y, Niksefat M (2018) Prevalence of type 1 and type 2 diabetes and its related factors in diabetic patients hospitalized in Khatam-ol-Anbia hospital in Shoushtar, 2014-15: A retrospective study. Journal of Research in Medical and Dental Science 6(3): 421-426.

2. Feyzi R, Madmoli M (2019) Epidemiologic approach to quality of life in diabetic patients: a systematic review study. IJMBS 3(5): 24-28.

3. Feyzi R, Madmoli M (2019) A systematic review with the epidemiological approach to the quality of life of cancer patients. IJMBS 3(5): 18-23.

4. Madmoli M, Madmoli M, Khodadadi M, Madmoli Y (2019) The communication Between Diabetes and Alzheimer's Disease: a study on diabetic patients referring to health centers in three cities of Khuzestan province. Int Res in Med Health Sci 2(2): 8-5.

5. Madmoli M, Madmoli M, Aliabad MA, Khodadadi M, Ahmadi FP (2019) A systematic review on the impact of empowerment in improving self-care behaviors and some other factors in diabetic patients. Int J Health Biol Sci 2(1): 11-16.

6. Madmoli M, Samsamipour M (2019) The Most Important Methods for Reducing Chemotherapy-Induced Nausea and Vomiting in Cancer Patients: A Systematic Review Study. Int Healthc Res J 3(1): 3-8.

7. Madmoli M, Khodadadi M, Ahmadi FP, Niksefat M (2019) A Systematic review on the impact of peer education on self-care behaviors of patients. International Journal of Health and Biological Sci 2(1): 1-5.

8. Madmoli M, Eilami O, Rezaie K, Aliabad MA, Moslemirad M (2018) Diabetes and the risk of suffering cardiovascular Diseases: A two-year retrospective study. International Journal of Ecosystems and Ecology Science (IJEES) 8(3): 649-656.

9. Madmoli M Rostami F, Mirsami Yazdi N, Mosavi A, Baraz Sh (2018) Evaluation of Prevalence of Diabetic Foot Ulcer and Its Related Factors in Diabetic Patients Admitted to KHatam-ol-Anbia Hospital in Shoushtar During 2015-2016: A Retrospective Study. IJEES 8(3): 545-552.

10. Rostami F, Madmoli M, Mirsami Yazdi N, Baraz Sh (2018) Evaluation of The Prevalence of Lower Limb Amputation and Its Related Factors in Diabetic Patients Admitted to KHatam-ol-Anbia Hospital in Shoushtar During The 2015-2016: A Retrospective Study. International Journal of Ecosystems and Ecology Science (IJEES) 8(3): 553-560.

11. Madmoli M, Madmoli Y, Mobarez F, Taqvaeinasab H, Darabiyan P, et al. (2019) Drugs abuse and increase in referral to hospital to prevent recurrence of diabetic foot ulcer infection. International Journal of Ayurvedic Medicine 10(1): 89-94.

12. Raisifar Z, Afshar Nia A, Madmoli M, Madmoli Y (2018) The Relationship Between Using Insulin and Suffering Alzheimer's Disease in Patients 
with Diabetes: A Two-Year Study. International Journal of Ecosystems and Ecology Science (IJEES) 8(3): 623-628.

13. Madmoli M, Izadi M, Madmoli M (2019) A Systematic Review Study on Interventions to Reduce Nausea and Vomiting Caused By Chemotherapy. Journal of Biotechnology and Bioengineering 3(1): 14-20.

14. Madmoli M, Izadi M, Khodadadi M, Khodadadi M, Papi Ahmadi F, Abbaszade Aliabad M (2019) A Systematic Review Study on the Effect of Multimedia Training on Self-Care Behaviors and Quality of Life in Diabetic Patients. Journal of Genetics and Genetic Engineering 2(4): 1-6.

15. Madmoli M, Izadi M, Madmoli M, Khodadadi M, Ahmadi FP, Aliabad MA (2019) A Systematic Review Study of the Most Important Complications of Chemotherapy in Cancer Patients. Journal of Biotechnology and Bioengineering 3(1): 2-7.

16. Raisifar Z, Afshar Nia A, Maghamesi Moarrefi H, Madmoli M (2018) Evaluation of Gi Bleeding Prevalence and Its Related Factors in Diabetic Patients Hospitalized in KHatam-ol-Anbia Hospital During 2015-16: A Retrospective Study. International Journal of Ecosystems and Ecology Science (IJEES) 8(3): 609-614.

17. Madmoli M, Kord Z, Bandani A, Sedighi N, Rezaei Shandiz M, Darabiyan P, AfsharNia A (2019) Epidemiological and clinical study of patients with Alzheimer's in Five Cities of Khuzestan Province in 2016-2018. Medical Science 23(95): 1-5.

18. Mashali H, Toleideh F, Rahmani R, Darabiyan P, Madmoli M (2018) The predictive role of Hyperlipidemia in the incidence of ACS in patients referring to Shahidzadeh Hospital in Behbahan in 2016 -2017. Medical Science 22(94): 566-570

19. Madmoli M, Modheji Y, Rafi A, Feyzi R, Darabiyan P, et al. (2019) Diabetes and its predictive role in the incidence of Alzheimer's disease. Medical Science 23(95): 30-34.

20. Madmoli M, Shaidaei MF, Rohani A, Madmoli Y, Khodadadi M (2019) Some predisposing factors and affecting cancer under the age of 35: A 6-year study on 2721 cancer patients. International Journal of Ayurvedic Medicine 10(1): 62-67.

21. Rafiee E, Khaledi M, Madmoli M, Zafari M, Lotfizadeh M (2019) The Correlation between Blood Pressure and BMI in Students of Shahrekord University of Medical Sciences in 2013-14. International Journal of Ayurvedic Medicine 10(1): 113-117.

22. Madmoli M, Saeidilandi M, Latifinasab R, Fatehimoghaddam S, Mobarez F, Darabiyan P (2019) Hypertension and Risk of Acute Coronary Syndrome (ACS) in Patients with ACS: A Study on 926 patients with ACS. International Journal of Ayurvedic Medicine 10(1): 22-26.

23. Madmoli M, Madmoli Y, Taqvaeinasab H, Khodadadi M, Darabiyan P, Rafi A (2019) Some influential factors on severity of diabetic foot ulcers and Predisposing of limb amputation: A 7-year study on diabetic patients. International Journal of Ayurvedic Medicine 10(1): 75-81.

24. Madmoli M, Abbaszade Aliabad M, Madmoli M, Khodadadi M, Papi Ahmadi F (2019) The Effect of Some Factors on Self-Care in Diabetic Patients: A Systematic Review. Journal of Genetics and Genetic Engineering 3(1): 21-25.

25. Madmoli M (2019) A Systematic Review Study on the Changer Factors of the Quality of life in Cancer Patients. Int Res Med Health Sci 2(1): 8-15.

26. Madmoli M (2019) A systematic Review Study on the Results of Empowerment-Based Interventions in Diabetic Patients. Int. Res. Med. Health Sci 2(1): 1-7.

27. Madmoli M, Fallah bagher shaidaei M, Rohani A, Darabiyan P, Mobarez F (2019) The correlation between alcohol consumption and reducing the age of cancer incidence in patients with this disease. Medical Science 23(95): 48-53.

28. Madmoli M, Mahmoudi Dehcheshmeh Z, Rafi A, Zahra Kord, Fariba Mobarez, et al. (2019) The rate of some complications and risk factors of diabetes in diabetic patients: Study on cases of 3218 diabetic patients. Medical Science 23(95): 63-68.
29. Mostafa Madmoli, Mehran Yarbig, Negin Sedighi, Pouriya Darabiyan, Fariba Mobarez (2019) Communication between body mass index and the risk of obesity-related cancer: A 5-year study on patients with cancer. Medical Science 23(95): 69-74.

30. Madmoli M (2018) Clinical and Laboratory Finding in Children with Leukemia: A Systematic Review. International Journal of Research Studies in Science, Engineering and Technology 5(10): 1-6.

31. Madmoli M (2018) Evaluation of Chemotherapy Complications in Patients with Cancer: A systematic Review. International Journal of Research Studies in Science, Engineering and Technology 5(12): 59-64.

32. Madmoli M, Madmoli Y, Khodadadi M, Samsamipour M (2019) Study of Some Effective Treatments for Accelerating Diabetic Foot Ulcer Healing: A Systematic Review. International Journal of Research Studies in Science, Engineering and Technology 6(2): 34-39.

33. Madmoli M, Madmoli Y, Khodadadi M, Samsamipour M (2019) Factors Affecting the Level of Glycosylated Hemoglobin in Patients with Diabetes: A Systematic Review. Annals of Microbiology and Infectious Diseases 2(1): 43-47.

34. Madmoli, M (2019) A Systematic Review Study on the Communication between Diabetes and Cancer: The Serious Risk of Cancer in Diabetic Patients. International Research in Medical and Health Sci 2(2): 1-7.

35. Madmoli M (2019) Quality of Life in Patients with Cancer and Some Factors Affecting it: A Systematic Review. International Journal of Research Studies in Science, Engineering and Technology 6(1): 1-7.

36. Madmoli M, Madmoli Y, Khodadadi M, Samsamipour M (2019) Some Factors Affecting Quality of Life in Patients with Diabetes: A systematic Review. Annals of Microbiology and Infectious Diseases 2(1): 26-30.

37. Gheisari Z, Beiranvand R, Karimi A, Ghalavandi S, Soleymani A, et al. (2018) Relationship between Occupational Stress and Cardiovascular Risk Factors Determination: A Case-control Study. Journal of Research in Medical and Dental Science 6(3): 287-293

38. Madmoli M, Nikpay S (2018) An Investigation of the Relationship between Spiritual Health and Depression, Anxiety, and Stress among Students of Ilam University of Medical Sciences. Journal of Research in Medical and Dental Science 6(3): 294-300

39. Adavi A, Hamid N, Attari Y, Madmoli Y, Madmoli M (2016) Study of the Effect of Problem-Solving Skills Training on Creactivity and Assertiveness Among High School Students. Iranian Journal of Nursing Res 11(5): 48-55.

40. Madmoli M, Madmoli Y, Bigdeli Shamloo MB, Etebari A, Mahmoodi Kouhi A, et al. (2017) The Relationship Between Depression and Religiousness in Female High School Students of Masjed Soleyman in 2015. Journal of Pediatric Nursing 3(4): 15-22.

41. Roughani A, Madmoli M, Raisifar Z, Kikhavani S, Yasemi M, et al. (2018) The prevalence of behavioral disorders and its related factors in elementary school children in Ilam City in 2011-2012. Indian Journal of Forensic Medicine \& Toxicology 12(4): 165-169.

42. Madmoli Y, Madmoli M, Qashqaei Nezhad N, Bosak S (2016) Prevalence of depression and associated factors in adolescents of Masjed-Soleyman. Iranian Journal of Pediatric Nursing 2(4): 22-26.

43. Madmoli Y, Aslani A, Ahmadi Y, Mousavi M, Mashalchi H, et al. (2017) Study Habits and Related Factors in Students of Nursing and Midwifery College of Dezful University of Medical Sciences in 2015. Iranian Journal of Nursing Research (IJNR).

44. Madmoli Y, Akhaghi Dezfuli SM, Beiranvand R, Saberi Pour B, Azami M, Madmoli M (2017) An epidemiological and clinical survey of patients with $\beta$-thalassemia in dezful in 2015. Iranian Journal of Epidemiology 13(2): 145-152

45. Madmoli Y, Salimi M, Madmoli M, Davoodi N, Rostami F, et al. (2019) The relationship between spiritual well-being and family cohesion in patients with thalassemia major. J Res Med Dent Sci 7(2): 203-209. 
46. Madmoli Y, Salimi M, Madmoli M, Maraghi E, Pelarak F, et al. (2019) The effect of orem self-care model on health-related quality of life of patients with thalassemia major. J Res Med Dent Sci 7(2): 170-176.

47. Madmoli Y, Madmoli M, Davoodi N, Niksefat M, Saberipour B, et al. (2019) Health related quality of life in patients with $\beta$-thalassemia major in northern Khuzestan Province, southwest of Iran in 2015-2016. J Res Med Dent Sci 7(2): 164-169.

48. Madmoli Y, Akhaghi Dezfuli SM, Adavi A, Maraaghi E, Heidari Soureshjan R, et al. (2018) The Effect of Orem Self-Care on Mental Health of Patients with Thalassemia Major. Journal of Clinical Nursing and Midwifery 7(2): 108-115.

49. Madmoli M, Madmoli Y, Rahmati P, Adavi A, Yousefi N, et al. (2017) Quality of Life and Some Related Factors in Patients with Beta Thalassemia Major in Southwest Iran. JCCNC 3(2): 139-146.

50. Madmoli Y, Beiranvand R, Korkini N, Mashalchi H, Karimi H (2016) Comparison of health-related quality of life in beta thalassemia major and healthy people in Dezful in 2015. Iran J Nurs Res 11(1): 9-16.

51. Wang JY, Chao TT, Lai CC, Wang CY, Wu VC, et al. (2013) Risk of colorectal cancer in type 2 diabetic patients: a population-based cohort study. Japanese journal of clinical oncology 43(3): 258-263.

52. Bosetti C, Rosato V, Polesel J, Levi F, Talamini R, et al. (2012) Diabetes mellitus and cancer risk in a network of case-control studies. Nutrition and cancer 64(5): 643-651.

53. Azizi H, Delpisheh A, Sayehmiri K, Asadollahi K, Esmaeili ED, et al. (2016) Risk of Colorectal Cancer in Type2 Diabetic Patients. Majallah-i pizishki-i Danishgah-i Ulum-i Pizishki va Khadamat-i Bihdashti-i Darmani-i Tabriz 38(4): 56.

54. Vigneri P, Francesco F, Laura S, Giusepp P, Richado V (2009) Diabetes and cancer. Endocr Relat Can 16: 1103-1123.

55. Eddi R, Karki A, Shah A, DeBari VA, DePasquale JR (2012) Association of type 2 diabetes and colon adenomas. Journal of gastrointestinal cancer 43(1): 87-92

56. Inoue M, Iwasaki M, Otani T, Sasazuki S, Noda M, et al. (2006) Diabetes mellitus and the risk of cancer: results from a large-scale population based cohort study in Japan. Archives intern med 166(17): 1871-1877.

\section{ISSN: 2574-1241}

DOI: 10.26717/BJSTR.2019.19.003313

Mohammad Madmoli. Biomed J Sci \& Tech Res

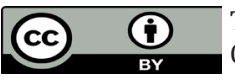

This work is licensed under Creative Commons Attribution 4.0 License

Submission Link: https://biomedres.us/submit-manuscript.php
57. La Vecchia C, Negri E, Decarli A, Franceschi S (1997) Diabetes mellitus and the risk of primary liver cancer. Int j can 73(2): 204-207.

58. Luo J, Chlebowski R, Liu S, McGlynn KA, Parekh N, White DL, Margolis KL (2013) Diabetes mellitus as a risk factor for gastrointestinal cancers among postmenopausal women. Cancer Causes Control 24(3): 577-85.

59. Rousseau MC, Parent MÉ, Pollak MN, Siemiatycki J (2006) Diabetes mellitus and cancer risk in a population-based case-controlstudy among men from Montreal, Canada. Int j can 118(8): 2105-2109.

60. Luo S, Li JY, Zhao LN, Yu T, Zhong W, et al. (2016) Diabetes mellitus increases the risk of colorectal neoplasia: an updated meta-analysis. Clin Res Hepatol Gastroenterol 40(1): 110-123.

61. Azizi H, Asadollahi KH, Davtalab Esmaeili E, Mirzapoor M (2015) Iranian Dietary Patterns and Risk of Colorectal Cancer. Health Promot Perspect 5(1): 72-80.

62. Ulaganathan V, Kandiah M, ZalilahMS, Faizal JA, Fijeraid H, et al. (2012) Colorectal Cancer and its Association with the Metabolic Syndrome: A Malaysian Multi-Centric Case-Control Study. Asian Pac J Cancer Prev 13(8): 3873-3877.

63. Hosseinzadeh A, Daraei A (2012) Environmental Factors Associated with Sporadic Colorectal Cancer. Esfahan. Iran J Health System Research 8(2): 229-236.

64. Maajani K, Khodadost M, Fattahi A, Shahrestanaki E, Pirouzi A, et al (2019) Survival Rate of Colorectal Cancer in Iran: A Systematic Review and Meta-Analysis. Asian Pacific Journal of Cancer Prevention. 20(1): $13-21$

65. Baghestani AR, Daneshvar T, Pourhoseingholi MA, Asadzade H (2014) Survival of colorectal cancer patients in the presence of competing risk. Asian Pac J Cancer Prev 15(15): 62-68.

66. Pourhoseingholi MA, Zali MR (2012) Colorectal cancer screening: Time for action in Iran. World J Gastrointest Oncol 4(4): 82-83.

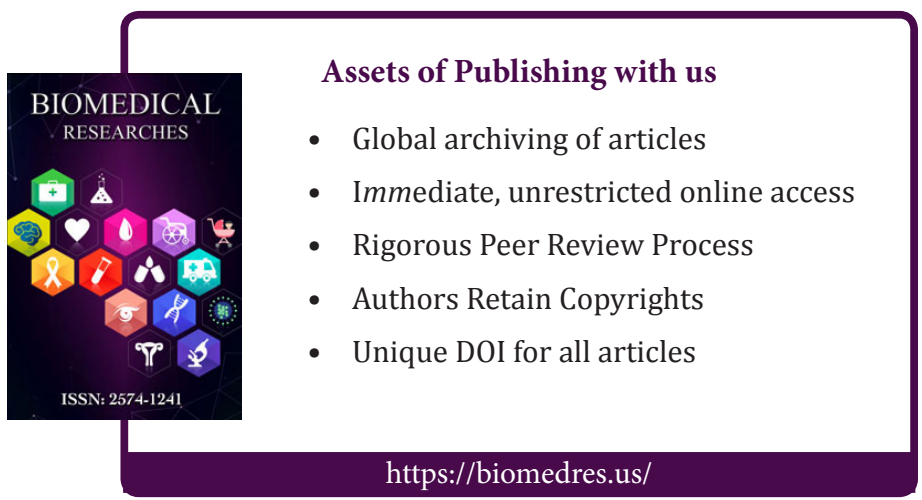

